\title{
Tariftreueregelungen in Deutschland - Ein aktueller Überblick
}

Seit das Bundesverfassungsgericht im Jahr 2006 in einem Grundsatzurteil die verfassungsrechtliche Zulässigkeit von Tariftreueregelungen bekräftigt hat, hat die politische Auseinandersetzung über dieses Thema wieder deutlich an Dynamik gewonnen. Immer mehr Bundesländer haben landesspezifische Vergabegesetze mit Tariftreueklauseln verabschiedet, wonach öffentliche Aufträge nur noch an solche Unternehmen vergeben werden dürfen, die sich an bestehende Tarifverträge halten. Hinzu kommt, dass sich die Tariftreuegesetze nicht mehr nur auf die Bauwirtschaft konzentrieren, sondern weitere Branchen einbeziehen. Schließlich sind die ersten Bundesländer dazu übergegangen, neben Tariftreueklauseln auch Mindestlohnregelungen in das Vergabegesetz aufzunehmen. Nach einem aktuellen Urteil des Europäischen Gerichtshofes von Anfang April 2008 ist die Zukunft der Tariftreuereglungen jedoch mehr als ungewiss.

\section{Einleitung}

Vor dem Hintergrund einer seit Mitte der 1990er Jahre rückläufigen Tarifbindung bilden Tariftreueregelungen ein ordnungspolitisches Instrument, um das Tarifvertragssystem in Deutschland zu stabilisieren. Der Staat nutzt hierbei seine Marktmacht als öffentlicher Auftraggeber, um Unternehmen zur Einhaltung von Tarifstandards zu bewegen. Ohne entsprechende Regelungen würde der Staat selbst zur weiteren Erosion des Tarifvertragssystems beitragen, da er normalerweise gezwungen ist, das günstigste Angebot anzunehmen und damit nicht-tarifgebundenen Unternehmen einen strukturellen Wettbewerbsvorteil einräumen müsste. Tariftreuegesetze verfolgen demnach das Ziel, bei der öffentlichen Vergabe gleiche Wettbewerbsbedingungen herzustellen, sodass die Konkurrenz nicht primär über die Lohn- und Arbeitskosten, sondern über die Qualität der Leistungen ausgetragen wird. Der vorliegende Beitrag liefert einen aktuellen Überblick über die bestehenden Tariftreueregelungen in Deutschland.

\section{Ökonomische Bedeutung des öffentlichen Auftragswesens}

In Deutschland geben die etwa 30.000 Vergabestellen des Bundes, der Länder und der Kommunen jährlich mehr als 360 Milliarden Euro für öffentliche Aufträge aus (BMWi 2007). Im Jahr 2005 entsprach dies etwa $16 \%$ des Bruttoinlandsproduktes (BIP). Damit bewegt sich Deutschland etwa im europäischen Durchschnitt (European Commission 2004). Eine noch größere ökonomische Bedeutung haben öffentliche Aufträge hingegen in Frankreich, Großbritannien, Schweden und Österreich sowie in den Niederlanden, wo das öffentliche Auftragswesen mit einem Anteil von $24 \%$ des BIP den mit Abstand höchsten Wert aufweist (Abbildung 1).

Die hohe ökonomische Bedeutung des öffentlichen Auftragswesens verleiht dem Staat als Nachfrager eine erhebliche Marktmacht und eröffnet ihm ein weitreichendes politisches Steuerungspotenzial (Handler 2005). Die Vergabe öffentlicher Aufträge bildet demnach in der Praxis ein wichtiges Instrument, um bestimmte wirtschaftspolitische Ziele, wie z. B. die Förderung technologischer Innovationen, die Unterstützung von kleineren und mittleren Unternehmen oder die Förderung regionaler Wirtschaftsstrukturen, durchzusetzen.

Umstritten ist jedoch, ob der Staat das öffentliche Auftragswesen auch zur Durchsetzung sozialer und ökologischer Ziele einsetzen soll. In einem aktuellen Gutachten zum öffentlichen Beschaffungswesen in Deutschland hat sich der Wissenschaftliche Beirat des Bundesministeriums für Wirtschaft (BMWi) dezidiert gegen die Berücksichtigung sogenannter „vergabefremder Ziele“ bei öffentlichen Aufträgen ausgesprochen, da diese „unweigerlich zu einer Vernachlässigung des Hauptziels wirtschaftlicher Einkauf" führt (BMWi 2007,
S. 9). Nach Ansicht des Beirates besteht bei einer stärker marktorientierten Einkaufspolitik ein Einsparungspotenzial von etwa $10 \%$, das die öffentlichen Haushalte erheblich entlasten würde (ebd., S. 5).

Gegen eine solche Position kann eingewendet werden, dass auch die NichtBerücksichtigung sozialer und ökologischer Kriterien mit vielfältigen öffentlichen Folgekosten verbunden ist, die das gewonnene Einsparpotenzial bei der öffentlichen Vergabe durch Mehrkosten an anderer Stelle wieder zunichte machen. So haben z. B. zahlreiche öffentliche Einrichtungen versucht, dadurch Kosten zu sparen, dass sie ihre Postdienstleitungen nicht mehr von der Deutschen Post, sondern von billigeren privaten Postfirmen erledigen lassen. Letztere können ihre kostengünstigeren Dienstleistungen jedoch oft nur dadurch anbieten, dass sie ihren Beschäftigten sehr niedrige Löhne zahlen, die dann wieder durch zusätzliche Sozialleistungen des Staates aufgestockt werden müssen (Butollo 2007, S. 7f.).

Thorsten Schulten, Dr., Wissenschaftler im WSI in der Hans-Böckler-Stiftung. Arbeitsschwerpunkte: Arbeits- und Tarifpolitik in Europa. e-mail: Thorsten-Schulten@boeckler.de Michael Pawicki studiert Sozia/wissenschaften an der Ruhr-Universität Bochum und hat im Rahmen eines zweimonatigen Praktikums im WSI zum Thema Tariftreue gearbeitet. e-mail: michael.pawicki@gmx.de 


\section{Abb. 1: Ausgaben für öffentliche Aufträge in \% des Bruttoinlands- produkts (BIP) 2005}

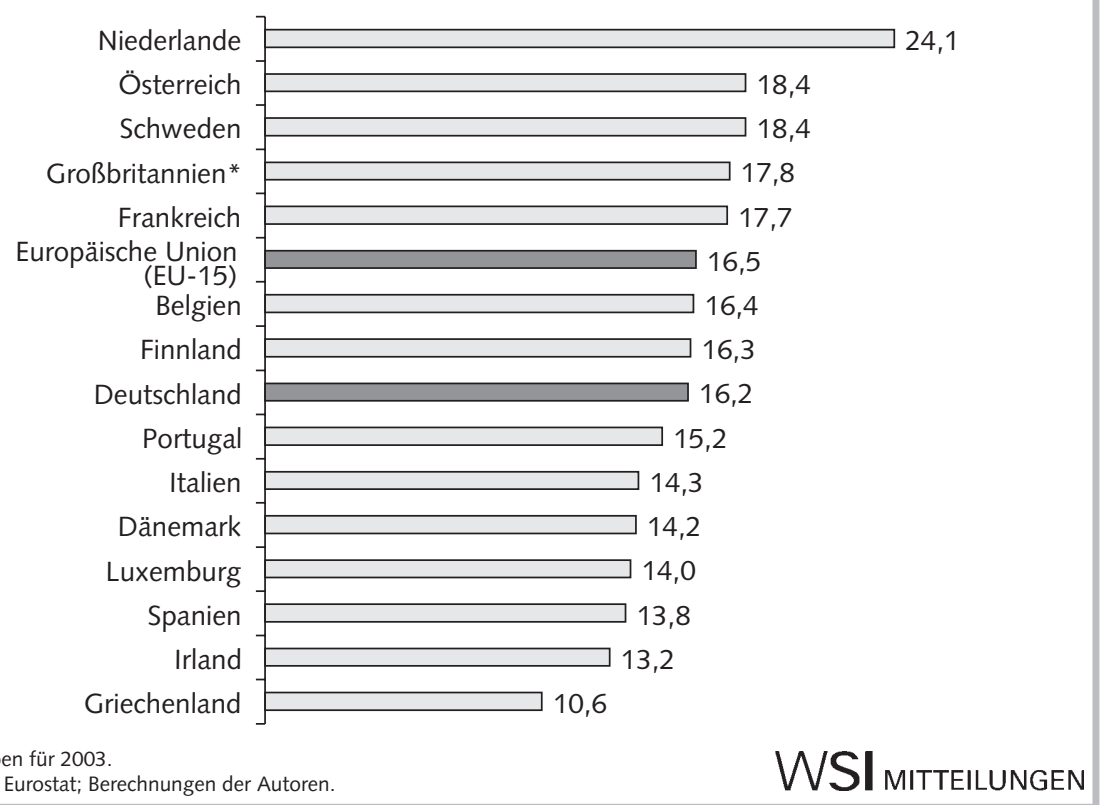

\section{3 \\ Rechtliche Rahmen- bedingungen für Tariftreuereglungen}

Bei der Koppelung öffentlicher Aufträge an die Einhaltung bestimmter sozialer Mindeststandards handelt es sich um eine international weit verbreitete Praxis, die sich historisch bis ins 19. Jahrhundert zurückverfolgen lässt (McCrudden 2004). Im Rahmen der Internationalen Arbeitsorganisation (IAO/ILO) wurde bereits 1949 ein „Übereinkommen über die Arbeitsklauseln in den von Behörden abgeschlossenen Verträgen" (IAO-Übereinkommen Nr. 94) verabschiedet, nach dem bei allen öffentlichen Aufträgen vertraglich sichergestellt werden soll, dass die den Auftrag ausführenden privaten Unternehmen bestehende gesetzlich und tarifvertraglich vorgeschriebene Lohnniveaus und Arbeitsstandards einhalten. Im Gegensatz zu zahlreichen anderen europäischen Ländern, wie z. B. Frankreich, Italien, Großbritannien, den Niederlanden und Österreich, hat Deutschland dieses Übereinkommen jedoch bis heute nicht ratifiziert.

Innerhalb Europas wird die öffentliche Auftragsvergabe heute in hohem Maße durch EU-Recht reguliert. Durch eine Vielzahl von EU-Richtlinien ist mittlerweile ein komplexes Regelwerk geschaffen worden. ${ }^{1}$
Die Berücksichtigung sozialer Ziele bei der Vergabe öffentlicher Aufträge wird dabei durch das EU-Recht ausdrücklich erlaubt (Bechtolsheim/Betz 2006, S. 6ff.). In einer eigens hierzu von der Europäischen Kommission (2001, S. 8) formulierten Mitteilung heißt es, dass "generell ... jeder Auftraggeber bei der Definition der Ware oder Dienstleistung, die er erwerben möchte, diejenigen Waren, Dienstleistungen oder Bauarbeiten wählen (kann) ..., die seinen sozial-politischen Anliegen entsprechen." In der aktuell gültigen Vergabe-Richtlinie der EU aus dem Jahr 2004 wird in Artikel 26 ausdrücklich darauf hingewiesen, dass öffentliche Auftraggeber die Möglichkeit haben, zusätzliche Bedingungen für die Ausführung des Auftrags vorzuschreiben. Explizit erwähnt werden hierbei „soziale und umweltbezogene Aspekte“ (Europäische Union 2004, S. 134).

Unabhängig von dem europäischen Vergaberecht gibt es eine kontroverse Debatte darüber, ob die Festlegung sozialer Vergabekriterien gegen das europäische Primärrecht - darunter insbesondere gegen die in Artikel 49 des EU-Vertrages garantierte Freiheit des Dienstleistungsverkehrs - verstößt (Bechtolsheim/Betz 2006, S. 39ff.). Nach einer Klage gegen das niedersächsische Tariftreuegesetz hat das Oberlandesgericht in Celle beim Europäischen Gerichtshof (EuGH) ein Vorabentscheidungsersuchen eingereicht, um die Frage zu klären, ob Tariftreueregelungen gegen die europäische Dienstleistungsfreiheit verstoßen. In den Schlussanträgen zu dem Verfahren kommt der Generalanwalt des EuGH zu dem Ergebnis, dass Vorschriften zur Tariftreue mit dem EU-Recht vereinbar sind (Bot 2007).

Entgegen der Meinung des Generalanwaltes hat der EuGH in seinem aktuellen Urteil vom 3. April 2008 jedoch die Position vertreten, dass Tariftreueregelungen mit der europäischen Dienstleitungsfreiheit nicht vereinbar sind (Europäischer Gerichtshof 2008). Nach Ansicht des EuGH darf Letztere zum Schutz der Beschäftigten nur durch solche Regelungen eingeschränkt werden, die durch die Europäische Entsenderichtlinie (Richtlinie 96/71/EG vom 16. Dezember 1996) abgedeckt sind, wozu namentlich gesetzliche Mindestlöhne und allgemeinverbindliche Tarifverträge gehören. Im Gegensatz hierzu sind Tariftreueregelungen nach dem EuGH durch die Entsenderichtlinie nicht gedeckt, da sie nur diejenigen Beschäftigten betreffen, die öffentliche Aufträge ausführen und somit keine universelle Gültigkeit haben.

In Deutschland unterliegt die Vergabe öffentlicher Aufträge einem komplexen rechtlichen Regelwerk, das verschiedene Gesetze umfasst (Leinemann 2007). Die allgemeinen Grundlagen finden sich im Gesetz gegen Wettbewerbsbeschränkungen (GWB, \$\$ 97-129), während die Einzelheiten zu den Verfahren der öffentlichen Auftragsvergabe in der Verordnung über die Vergabe öffentlicher Aufträge ( $\mathrm{VgV})$ sowie in den Verdingungsordnungen für Bauleistungen (VOB), für Lieferungen und Dienstleistungen (VOL) und für freiberufliche Leistungen (VOF) geregelt werden. Hinzu kommen die auf Landesebene existierenden Vergabegesetze.

Nach dem GWB müssen öffentliche Aufträge „an fachkundige, leistungsfähige und zuverlässige Unternehmen vergeben“ werden, wobei dasjenige Unternehmen den Zuschlag erhält, das das „wirtschaftlichste Angebot" unterbreitet (\$97, Abs. 4-5). Die bundesweiten Vergabegesetze enthalten selbst keine Bestimmungen über soziale Vergabekriterien. Das GWB schreibt ledig-

\footnotetext{
Die Europäische Kommission hat eine eigene Webpage zur öffentlichen Auftragsvergabe eingerichtet, auf der alle wichtigen EU-Dokumente heruntergeladen werden können (http://ec.europa.eu/ internal market/publicprocurement/legislation de.htm).
} 
lich vor, dass „andere oder weitergehende Anforderungen ... an Auftragnehmer nur gestellt werden (dürfen), wenn dies durch Bundes- oder Landesgesetz vorgesehen ist " (\$97, Abs. 4). Dies bildet die rechtliche Grundlage für Vergabegesetze auf Landesebene, einschließlich der darin enthaltenden Regelungen zur Tariftreue.

Im Juli 2006 hat darüber hinaus das Bundesverfassungsgericht (2006) ein Grundsatzurteil zur verfassungsrechtlichen Zulässigkeit von Tariftreueregelungen gefällt. Anlass war ein Vorlagebeschluss des Bundesgerichtshofs, der das Berliner Vergabegesetz für verfassungswidrig erklärt hatte, da es gegen die im Grundgesetz (Art.9) geschützte negative Koalitionsfreiheit verstoßen würde. Das Bundesverfassungsgericht hat diese Sichtweise zurückgewiesen und ausdrücklich die Verfassungskonformität von Tariftreueregelungen betont. Gemäß diesem Grundsatzurteil verfolgt der Gesetzgeber mit der Tariftreuereglung eine Reihe verfassungsrechtlich legitimer Ziele, darunter

- die Verhinderung eines „Verdrängungswettbewerbs über die Lohnkosten“,

- die „Bekämpfung der Arbeitslosigkeit“, - den „Schutz der Beschäftigung solcher Arbeitnehmer, die bei tarifgebundenen Unternehmen arbeiten",

- die „Erhaltung als wünschenswert angesehener sozialer Standards",

- die Entlastung der Systeme der sozialen Sicherheit und

- die Unterstützung des „Tarifvertragssystems als Mittel zur Sicherung sozialer Standards" (Bundesverfassungsgericht 2006, RN 86ff.).

Bezogen auf den letzten Punkt betont das Bundesverfassungsgericht ausdrücklich, dass ,der Gesetzgeber die Ordnungsfunktion der Tarifverträge unterstützen (darf), indem er Regelungen schafft, die bewirken, dass die von den Tarifparteien ausgehandelten Löhne und Gehälter auch für Nichtverbandsmitglieder mittelbar zur Anwendung kommen. Dadurch wird die von Art. 9 Abs. 3 GG intendierte, im öffentlichen Interesse liegende autonome Ordnung des Arbeitslebens durch Koalitionen abgestützt, indem den Tarifentgelten zu größerer Durchsetzungskraft verholfen wird“ (ebd., RN 90).

\begin{tabular}{|c|c|c|c|}
\hline \multicolumn{4}{|c|}{ Übersicht 1: Tariftreueregelungen in Deutschland } \\
\hline Bundesland & Gesetz & verabschiedet am & Geltungsbereich \\
\hline Bayern & $\begin{array}{l}\text { Gesetz über die Vergabe von } \\
\text { Bauaufträgen im Freistaat } \\
\text { Bayern (BayBauVG) }\end{array}$ & 28. Juni 2000 & Bauleistungen \\
\hline \multirow[t]{2}{*}{ Berlin } & $\begin{array}{l}\text { Berliner Vergabegesetz } \\
\text { (Vgb Bln) }\end{array}$ & 9. Juli 1999 & $\begin{array}{l}\text { Bauleistungen, } \\
\text { Dienstleistungen bei } \\
\text { Gebäuden und Immobilien }\end{array}$ \\
\hline & $\begin{array}{l}\text { Neufassung des Berliner } \\
\text { Vergabegesetzes }\end{array}$ & 13. März 2008 & $\begin{array}{l}\text { Sämtliche öffentliche } \\
\text { Aufträge }\end{array}$ \\
\hline \multirow[t]{2}{*}{ Bremen } & $\begin{array}{l}\text { Vergabegesetz für das Land } \\
\text { Bremen }\end{array}$ & 17. Dezember 2002 & Bauleistungen und ÖPNV \\
\hline & $\begin{array}{l}\text { Entwurf von SPD und } \\
\text { Grüne zur Revision des } \\
\text { Vergabegesetzes }\end{array}$ & $\begin{array}{l}\text { vorrausichtlich im } \\
\text { Mai } 2008\end{array}$ & $\begin{array}{l}\text { Bauleistungen, } \\
\text { Alle Dienstleistungs- } \\
\text { aufträge }\end{array}$ \\
\hline Hamburg & $\begin{array}{l}\text { Hamburgisches } \\
\text { Vergabegesetz (HmbVgG) }\end{array}$ & $\begin{array}{l}\text { 1. April } 2004 \\
\text { (rev. 13. Februar 2006) }\end{array}$ & Bauleistungen und ÖPNV \\
\hline Hessen & $\begin{array}{l}\text { Hessisches Vergabegesetz - } \\
(\mathrm{HVgG})\end{array}$ & 17. Dezember 2007 & $\begin{array}{l}\text { Bauleistungen, } \\
\text { Gebäudereinigerhandwerk, } \\
\text { Bewachungsgewerbe }\end{array}$ \\
\hline Niedersachsen & $\begin{array}{l}\text { Landesvergabegesetz } \\
\text { (LVergabeG) }\end{array}$ & $\begin{array}{l}\text { 2. September } 2002 \\
\text { (rev. 9. Dezember 2005) }\end{array}$ & $\begin{array}{l}\text { Bauleistungen } \\
\text { (bis zur Revision } 2005 \\
\text { auch ÖPNV) }\end{array}$ \\
\hline Rheinland-Pfalz & $\begin{array}{l}\text { Entwurf der Landesregierung } \\
\text { für ein Landestariftreuegesetz } \\
\text { (LTTG) }\end{array}$ & $\begin{array}{l}\text { vorrausichtlich im } \\
\text { Juni } 2008\end{array}$ & $\begin{array}{l}\text { Bauleistungen, } \\
\text { Gebäudereinigerhandwerk, } \\
\text { Bewachungsgewerbe, } \\
\text { Gebäude und } \\
\text { Immobilienwirtschaft } \\
\text { Abfallentsorgung, ÖPNV }\end{array}$ \\
\hline Saarland & $\begin{array}{l}\text { Saarländische Bauaufträge- } \\
\text { Vergabegesetz (SaarBauVG) }\end{array}$ & 23. August 2000 & Bauleistungen \\
\hline $\begin{array}{l}\text { Schleswig- } \\
\text { Holstein }\end{array}$ & $\begin{array}{l}\text { Gesetz zur tariflichen } \\
\text { Entlohnung bei öffentlichen } \\
\text { Aufträgen (Tariftreuegesetz) }\end{array}$ & 7. März 2003 & $\begin{array}{l}\text { Bauleistungen, } \\
\text { ÖPNV, } \\
\text { Abfallentsorgung }\end{array}$ \\
\hline \multicolumn{4}{|c|}{ Wieder aufgehobene Tariftreuegesetze: } \\
\hline $\begin{array}{l}\text { Nordrhein- } \\
\text { Westfalen }\end{array}$ & $\begin{array}{l}\text { Tariftreuegesetz Nordrhein- } \\
\text { Westfalen (TariftG NRW) }\end{array}$ & $\begin{array}{l}\text { 17. Dezember } 2002 \\
\text { aufgehoben zum } \\
\text { 21. November } 2006\end{array}$ & Bauleistungen und ÖPNV \\
\hline $\begin{array}{l}\text { Sachsen- } \\
\text { Anhalt }\end{array}$ & $\begin{array}{l}\text { Vergabegesetz Sachsen- } \\
\text { Anhalt (VergabeG LSA) }\end{array}$ & $\begin{array}{l}\text { 29. Juni } 2001 \\
\text { aufgehoben zum } \\
\text { 13. August } 2002\end{array}$ & Bauleistungen \\
\hline \multicolumn{3}{|c|}{ Quelle: WSI-Tarifarchiv 2008.} & WSI MitTEILUNGEN \\
\hline
\end{tabular}

\subsection{CHRONOLOGIE DER EREIGNISSE}

\section{Tariftreueregelungen in Deutschland}

Im Frühjahr 2008 verfügen acht von 16 Bundesländern über eigene Vergabegesetze mit Regelungen zur Tariftreue (Übersicht 1). Mit der geplanten Verabschiedung eines Landesvergabegesetzes in Rheinland-Pfalz, die vorrausichtlich im Juni 2008 stattfinden wird, wird sich die Zahl auf neun Länder erhöhen. Auffällig ist hierbei ein deutliches Ost-West-Gefälle; in Westdeutschland fehlen lediglich in zwei Ländern (BadenWürttemberg und Nordrhein-Westfalen), in Ostdeutschland hingegen in allen fünf Ländern Tariftreuegesetze. In zwei Bundesländern (Sachsen-Anhalt und NRW) wurden zudem in den letzten Jahren bestehende Tariftreueregelungen nach einem Regierungswechsel von einer SPD- zu einer CDU-geführten Landesregierung wieder abgeschafft.
Die Diskussionen um Tariftreuegesetze entwickelten sich in der zweiten Hälfte der 1990er Jahre vor dem Hintergrund einer rückläufigen Tarifbindung und zunehmender Arbeitslosigkeit. 1999 verabschiedete das seinerzeit von einer großen Koalition aus CDU und SPD regierte Berlin als erstes Bundesland ein Tariftreuegesetz. Ein Jahr später folgten die CDU- bzw. CSU-regierten Länder Bayern und Saarland mit eigenen Tariftreueregelungen. 2001 beschloss das sozialdemokratisch regierte Sachsen-Anhalt als erstes ostdeutsches Bundesland ein eigenes Tariftreuegesetz. Schließlich legte im Jahr 2002 die damalige rot-grüne Bundesregierung einen Entwurf für ein bundesweites Tariftreuegesetz vor, der jedoch von einer Mehrheit der CDU-regierten Länder im Bundesrat abgelehnt wurde. Ausschlaggebend war hierbei der zuvor erfolgte Regierungswechsel in Sachsen-Anhalt, der die Mehrheitsverhältnisse im Bundesrat grundle- 


\begin{tabular}{|c|c|}
\hline \multicolumn{2}{|c|}{$\begin{array}{l}\text { Übersicht 2: Schwellenwerte für die Anwendung von Tariftreue- } \\
\text { regelungen }\end{array}$} \\
\hline Bundesland & Schwellenwerte - in Euro - \\
\hline Hessen & ab einem Auftragswert von 50.000 \\
\hline Niedersachsen & ab einem Auftragswert von 30.000 \\
\hline Rheinland-Pfalz & ab einem Auftragswert von 20.000 \\
\hline Bremen, Schleswig-Holstein & ab einem Auftragswert von 10.000 \\
\hline Bayern, Berlin, Hamburg, Saarland & kein Schwellenwert \\
\hline
\end{tabular}

gend veränderte. Zugleich wurde das Tariftreuegesetz in Sachsen-Anhalt nur ein Jahr nach seiner Verabschiedung wieder aufgehoben.

Nach dem Scheitern eines bundesweit gültigen Tariftreuegesetzes kam es zunächst in Bremen, Niedersachsen und NRW (2002) und dann in Schleswig-Holstein (2003) und in Hamburg (2004) zur Einführung landesspezifischer Tariftreueregelungen, wobei das Tariftreuegesetz in NRW nach der Übernahe der Landesregierung durch die CDU im Jahr 2006 wieder aufgehoben wurde. Nachdem das Bundesverfassungsgericht die verfassungsmäßige Zulässigkeit von Tariftreuereglungen bestätigt hatte, hat die politische Auseinandersetzung um dieses Thema noch einmal deutlich an Dynamik gewonnen. Sichtbarstes Zeichen hierfür ist die Einführung von Tariftreuegesetzen im christdemokratisch regierten Hessen (2007) und im sozialdemokratisch regierten Rheinland-Pfalz (2008). In allen übrigen Ländern haben die jeweiligen Oppositionsparteien konkrete Entwürfe für Tariftreuegesetze vorgelegt und damit das Thema auf die politische Tagesordnung gesetzt. Im März 2008 hat die schwarz-rote Landesregierung von Mecklenburg-Vorpommern angekündigt, bis zur Sommerpause 2008 einen Entwurf für ein Tariftreuegesetz vorzulegen. Weitere Länder könnten in Zukunft folgen. Schließlich haben der rot-rote Senat in Berlin und der rot-grüne Senat in Bremen eine weitreichende Revision ihrer bestehenden Gesetze beschlossen, die den Geltungs- und Regelungsbereich deutlich ausdehnen.

\subsection{GELTUNGSBEREICH DER TARIFTREUEREGELUNGEN}

Die in den verschiedenen Bundesländern bestehenden Tariftreueregelungen weisen bezogen auf ihren konkreten Geltungsbereich sehr große Unterschiede auf (Übersicht 1), wobei sich im Laufe der Jahre als klare Tendenz durchzusetzen scheint, die Reichweite immer weiter auszudehnen. So waren die ersten Tariftreuegesetze in Berlin, Bayern und dem Saarland im Wesentlichen auf die Bauindustrie beschränkt, deren tiefe ökonomische Strukturkrise in der zweiten Hälfte der 1990er Jahren den Ausgangspunkt der gesamten Tariftreuediskussionen bildete. Als zweiter Schritt wurde von einigen Bundesländern der öffentliche Nahverkehr mit in den Geltungsbereich der Tariftreuegesetze aufgenommen. Hintergrund war die forcierte Liberalisierungspolitik in diesem Sektor, die tarifgebundene Verkehrsbetriebe insbesondere bei öffentlichen Ausschreibungen systematisch gegenüber ihren tarif-ungebundenen Konkurrenten benachteiligt hätte. Ähnliches gilt auch für Branchen wie die Abwerk oder das Bewachungsgewerbe, die in die neueren Tariftreuegesetze mit aufgenommen wurden. Eine neue Qualität weisen schließlich die novellierten Tariftreuegesetze in den Stadtstaaten Bremen und Berlin auf, die generell für alle Dienstleistungsaufträge (Bremen) oder gar für sämtliche öffentliche Aufträge (Berlin) gelten sollen.

Ein wichtiger Unterschied im Geltungsbereich der verschiedenen Tariftreuegesetze liegt nicht nur in ihrer sektoralen, sondern auch in ihrer funktionalen Reichweite. In der Mehrzahl der Bundesländer sind die Tariftreueregelungen sowohl für das Land als auch für die Kommunen gleichermaßen verbindlich. In den Ländern Bayern, Saarland und Schleswig-Holstein gilt diese Verpflichtung jedoch nur für die Landesebene, während es den Kommunen frei steht, ob sie die Tariftreueregelungen anwenden.

Die tatsächliche Reichweite wird schließlich nicht unerheblich dadurch beeinflusst, ob in den Vergabegesetzen ein bestimmter Schwellenwert festgelegt ist, unterhalb dessen öffentliche Aufträge von der Tariftreueverpflichtung ausgenommen werden (Überfallentsorgung, das Gebäudereinigerhand- sicht 2). In fünf Bundesländern sind solche Schwellenwerte festgelegt worden, die sich zwischen einem Auftragsvolumen von $10.000 €$ in Bremen und Schleswig-Holstein und $50.000 €$ in Hessen bewegen. Der besonders hohe Schwellenwert in Hessen führt dazu, dass eine große Anzahl öffentlicher Aufträge von vornherein durch die Tariftreueregelungen nicht erfasst wird. Demgegenüber haben mit Bayern, Berlin, Hamburg und dem Saarland vier Bundesländer ganz auf die Festlegung von Schwellenwerten verzichtet.

\subsection{KONTROLLEN UND SANKTIONSMÖGLICHKEITEN}

Von herausragender Bedeutung für die Umsetzung der Tariftreueregelungen sind die in den Vergabegesetzen vorgesehenen Kontrollen und Sanktionsmöglichkeiten. In allen Bundesländern ist der öffentliche Auftraggeber berechtigt, bei den beauftragten Unternehmen Kontrollen durchzuführen und Einsicht in die entsprechenden Unterlagen zu nehmen. Im Allgemeinen gilt auch, dass der Hauptauftragnehmer für die Einhaltung der Tariftreueregelungen bei Subunternehmen verantwortlich ist und diese zu kontrollieren hat. In Bremen, Hamburg und Niedersachsen ist der öffentliche Auftraggeber zudem bei der Auswahl von Subunternehmen zustimmungspflichtig. In den vier norddeutschen Bundesländern (Bremen, Hamburg, Niedersachsen und SchleswigHolstein) ist darüber hinaus eine, ,verpflichtende Überprüfung" des Angebotes vorgesehen, wenn dieses um mindestens $10 \%$ vom nächst höheren Angebot abweicht.

Bei den Strafen und Sanktionsmöglichkeiten, die für Verstöße gegen die Tariftreueregelung vorgesehen sind, existieren zwischen den Bundesländern erhebliche Unterschiede (Übersicht 3). Zunächst gibt es in allen neun Bundesländern die Möglichkeit, Unternehmen bei Verstößen gegen die Tariftreue von zukünftigen Vergabeverfahren auszuschließen. In den meisten Ländern ist hierbei ein Zeitraum von „bis zu drei Jahren" vorgesehen. Allerdings haben lediglich Berlin und Hessen diese Sanktionsmöglichkeit als verpflichtende „Soll-Regel“ eingeführt, während alle übrigen Länder hier nur eine „Kann-Bestimmung“ festgelegt haben. In fünf Bundesländern besteht außerdem die Möglichkeit, bei Verstößen den Vertrag mit dem Auftragnehmer fristlos zu kündigen.

Von der Möglichkeit, bei Verstößen gegen die Tariftreue eine Vertragsstrafe aus- 
zusprechen, kann in sechs Bundesländern Gebrauch gemacht werden. Lediglich in Bayern, im Saarland und in Berlin wird auf die Ankündigung von Vertragsstrafen in den Vergabegesetzen verzichtet. Bei schuldhaften Verstößen der Auftragnehmer wird in der Regel eine Vertragsstrafe von $1 \%$ des Auftragswertes genannt. Bei mehreren Verstößen kann diese Vertragsstrafe in einigen Bundesländern auf bis zu $10 \%$ des Auftragswerts erhöht werden.

\section{Tariftreue in der Praxis - Evaluationsergebnisse NRW und Hamburg}

Obwohl einige Bundesländer nun schon seit geraumer Zeit über Tariftreuegesetze verfügen, ist über deren praktische Umsetzung nach wie vor nur sehr wenig bekannt. Bislang haben lediglich zwei Bundesländer die Vergabepraxis im Hinblick auf die Tariftreueregelungen evaluiert. Aus NRW (Stefaniak/Vollmer 2005) und Hamburg (Hamburger Senat 2007) liegen entsprechende Evaluierungsberichte vor, die auf Befragungen von Unternehmen und Vergabestellen beruhen und sich im Wesentlichen auf die Bauwirtschaft beziehen.

Beide Studien kommen zunächst zu dem Ergebnis, dass die große Mehrheit der betroffenen Unternehmen die Regelungen zur Tariftreue begrüßt und als ein sinnvolles ordnungspolitisches Instrument zur Bekämpfung von Schmutzkonkurrenz ansieht. In NRW erklärten 84 \% der Unternehmen, dass sie die Zielsetzungen des Tariftreuegesetzes grundsätzlich unterstützen (Stefaniak/Vollmer 2005, S. 35). ${ }^{2}$ Eine noch größere Unterstützung erzielte das Tariftreuegesetz bei den Unternehmen in Hamburg (Abbildung 2). Nur knapp $3 \%$ aller Unternehmen halten hier eine Tariftreueregelung nicht für sinnvoll. Knapp $80 \%$ geben stattdessen an, dass ihr Unternehmen direkt von der Tariftreueregelung profitiert hat. Entgegen der Argumentation von Tariftreuekritikern erklären $90 \%$ der Hamburger Bauunternehmen, dass sie durch die Tariftreueregelung keinen signifikant höheren Aufwand haben.

Der Evaluierungsbericht aus NRW kommt allerdings zu dem Ergebnis, dass es bei der Kontrolle der Tariftreue erhebliche Defizite gab. So gaben knapp 80 \% der 139 in NRW befragten Vergabestellen an, dass

\begin{tabular}{|c|c|c|c|c|c|}
\hline \multicolumn{6}{|c|}{$\begin{array}{l}\text { Übersicht 3: Sanktionsmöglichkeiten beim Verstoß gegen Tariftreue- } \\
\text { regelungen }\end{array}$} \\
\hline \multicolumn{2}{|c|}{$\begin{array}{c}\text { Ausschluss von } \\
\text { Vergabeverfahren }\end{array}$} & \multicolumn{2}{|c|}{$\begin{array}{c}\text { Fristlose Kündigung } \\
\text { des Auftrages }\end{array}$} & \multicolumn{2}{|c|}{ Vertragsstrafen } \\
\hline $\begin{array}{l}\text { bis zu } \\
3 \text { Jahren }\end{array}$ & $\begin{array}{l}\text { Bayern, Berlin, } \\
\text { Hessen, } \\
\text { Rheinland-Pfalz, } \\
\text { Saarland, } \\
\text { Schleswig- } \\
\text { Holstein }\end{array}$ & vorgesehen & $\begin{array}{l}\text { Bremen, } \\
\text { Hamburg, } \\
\text { Niedersachsen, } \\
\text { Rheinland-Pfalz, } \\
\text { Schleswig- } \\
\text { Holstein }\end{array}$ & $\begin{array}{l}\text { Für jeden schuldhaften } \\
\text { Verstoß: Vertragsstrafe } \\
\text { von } 1 \% \text { des } \\
\text { Auftragswertes }\end{array}$ & $\begin{array}{l}\text { Bremen, } \\
\text { Hamburg, } \\
\text { Niedersachsen, } \\
\text { Rheinland-Pfalz, } \\
\text { Schleswig- } \\
\text { Holstein }\end{array}$ \\
\hline $\begin{array}{l}\text { bis zu } \\
\text { einem } \\
\text { Jahr }\end{array}$ & $\begin{array}{l}\text { Bremen, } \\
\text { Niedersachsen }\end{array}$ & $\begin{array}{l}\text { nicht } \\
\text { vorgesehen }\end{array}$ & $\begin{array}{l}\text { Bayern, Berlin, } \\
\text { Saarland }\end{array}$ & $\begin{array}{l}\text { Bei mehreren } \\
\text { Verstößen: Vertrags- } \\
\text { strafe bis } 10 \% \text { des } \\
\text { Auftragswertes }\end{array}$ & $\begin{array}{l}\text { Niedersachsen, } \\
\text { Rheinland-Pfalz, } \\
\text { Schleswig- } \\
\text { Holstein }\end{array}$ \\
\hline $\begin{array}{l}\text { keine } \\
\text { zeitliche } \\
\text { Angabe }\end{array}$ & Hamburg & & & $\begin{array}{l}\text { Bei mehreren } \\
\text { Verstößen: Vertrags- } \\
\text { strafe bis zu } 5 \% \text { des } \\
\text { Auftragswertes }\end{array}$ & Hamburg \\
\hline
\end{tabular}

Abb. 2: Bewertung des Hamburger Vergabegesetzes durch die Unternehmen der Bauwirtschaft - in \%* -

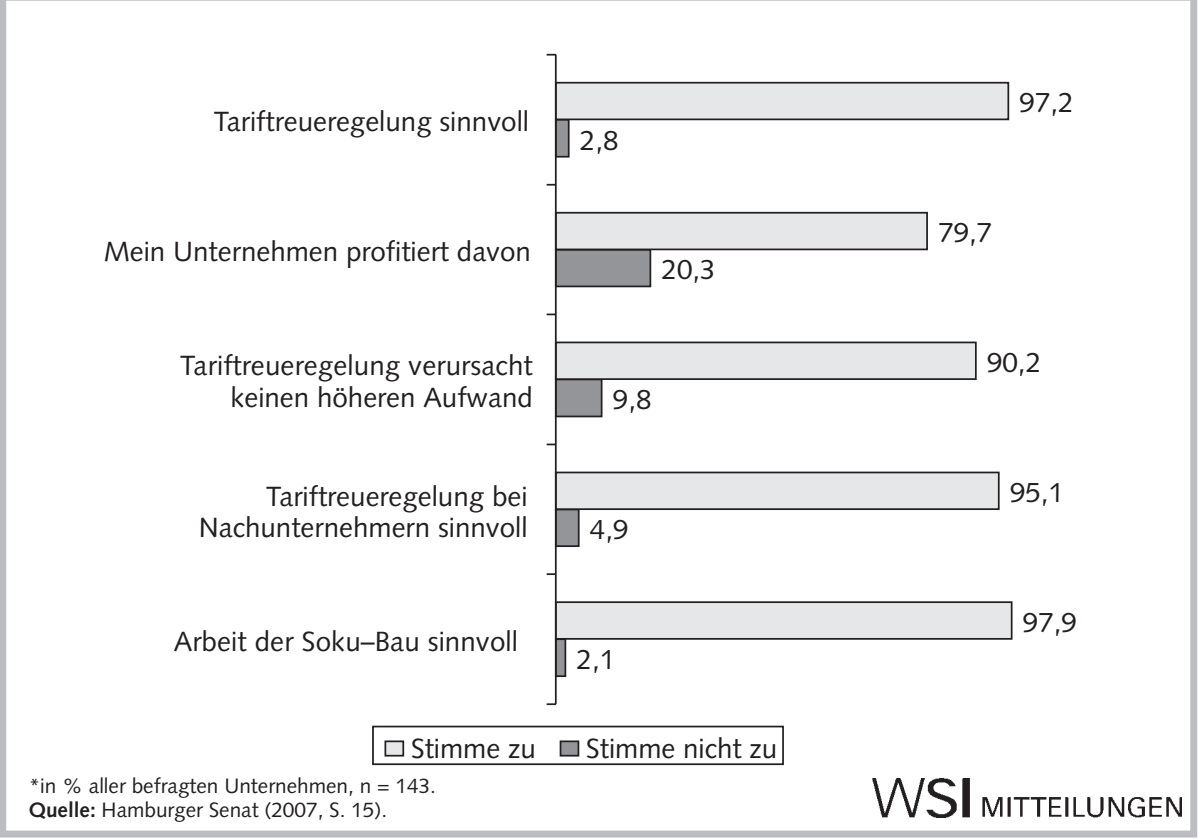

sie die Einhaltung der Tariftreue nicht kontrollieren (Stefaniak/Vollmer 2005, S. 31). Demgegenüber erklärten nur $34 \%$ der befragten Unternehmen, dass ihre Kalkulationen von den Vergabestellen überprüft wurden (ebenda, S. 42). Eine wesentliche Ursache für die geringe Kontrolltätigkeit sahen die Vergabestellen in fehlendem und nicht ausreichend qualifiziertem Personal (ebd., S. 27).

Vollkommen andersartige Erfahrungen wurden im Hinblick auf die Kontrolle der Tariftreue in Hamburg gemacht. Nach der Verabschiedung des Tariftreuegesetzes wurde bei der Hamburger Behörde für Stadtentwicklung und Umwelt ein eigenes Referat für „Tariflohn- und Nachunternehmerkontrolle" eingerichtet. Diese auch als „Soko-Bau“ bezeichnete Dienststelle ver- fügt über zehn eigene Beschäftigte und hat die Aufgabe, direkt vor Ort an den Baustellen die Lohnunterlagen $\mathrm{zu}$ überprüfen (Hamburger Senat 2007, S. 12). Mit dieser bundesweit einmaligen Kontrollstelle zur Überprüfung von Tariftreueregelungen ist es in Hamburg gelungen, eine hohe Kontrolldichte sicherzustellen und zugleich eine hohe Akzeptanz bei den betroffenen Unternehmen zu erzielen (ebd., S. 14).

Das Hamburger Beispiel deutet darauf hin, dass eine effiziente Umsetzung und Kontrolle von Tariftreueregelungen nicht

2 Insofern ist es recht verwunderlich, dass die gegenwärtige CDU/FDP-Landesregierung in NRW die Abschaffung des Tariftreuegesetzes ausgerechnet mit den Ergebnissen dieses Evaluierungsberichtes gerechtfertigt hat. 
allein den Vergabestellen überlassen werden darf, sondern diese durch eine zentrale Stelle auf Landesebene unterstützt werden sollten. In diesem Sinne wird z. B. in dem Entwurf für eine Neufassung des Bremer Vergabegesetzes die Einrichtung einer „zentralen Tariftreuestelle" vorgeschlagen (Fraktionen der SPD und Bündnis 90/Die Grünen in der Bremer Bürgerschaft 2007, S. 5). Auch in Berlin hat die Linke bei der Revision des Vergabegesetzes die Einrichtung einer unabhängigen Kontrollstelle vorgeschlagen, was jedoch bislang von der SPD mit Hinweis auf die zusätzlich entstehenden Kosten abgelehnt wird (Die Welt vom 10. März 2008).

\section{Tariftreue und Mindestlöhne}

Die Berücksichtigung der Zahlung bestimmter Mindestlöhne bei der Vergabe öffentlicher Aufträge gehört international zu den ältesten sozialen Vergabekriterien. Im Zuge der sogenannten Living Wages-Kampagnen haben diese Lohnstandards seit den 1990er Jahren insbesondere in den USA eine weite Verbreitung gefunden (Pollin et al. 2008). Bei den Living Wages handelt es sich um örtlich festgelegte Mindestlöhne, die in der Regel über den allgemeinen gesetzlichen Mindestlöhnen liegen und die besonderen örtlichen Lebenshaltungskosten berücksichtigen sollen. Sie sind für alle öffentlichen Einrichtungen sowie für private Unternehmen bei der Ausführung öffentlicher Aufträge verbindlich. In Europa finden sich Living Wage-Vereinbarungen insbesondere in Großbritannien. Eine Vorreiterrolle hat hierbei die Stadt London eingenommen, wo in den letzten Jahren unter Bürgermeister Ken Livingstone ein unfangreiches Regelungssystem zur Festsetzung von Living Wages eingeführt wurde (Burgess 2006; Greater London Authority 2007).

Tariftreueregelungen gehen zunächst deutlich über die Festlegung von Mindestlöhnen hinaus, da sie nicht nur die jeweils unterste Lohngruppe, sondern die gesamte durch den Tarifvertrag definierte Lohnstruktur als verbindliches Vergabekriterium festschreiben. Allerdings besteht in Deutschland seit geraumer Zeit das Problem, dass in zahlreichen Branchen in den unteren Lohngruppen extrem niedrige Ta- riflöhne festgelegt wurden, die kein existenzsicherndes Einkommen gewährleisten (Bispinck/Schulten 2008).

Vor diesem Hintergrund haben die Bundesländer Berlin und Bremen sich dafür entschieden, bei der Neufassung ihrer Vergabegesetze zusätzlich zur Tariftreue auch Mindestlohnregelungen aufzunehmen. In dem Entwurf zur Revision des Bremer Vergabegesetzes heißt es hierzu, dass „Aufträge des Landes und der Gemeinden, für die kein maßgeblicher Tarifvertrag ... angegeben werden kann, ... nur an solche Unternehmen vergeben werden (dürfen), die sich bei der Angebotsabgabe schriftlich verpflichten, ihren am Ort der Leistung eingesetzten Arbeitnehmerinnen und Arbeitnehmern bei der Ausführung dieser Leistungen mindestens ein Entgelt in Höhe des tarifvertraglich vorgesehenen Mindestentgelts für Arbeitnehmerinnen und Arbeitnehmer des Landes und der Gemeinden zu bezahlen" (Fraktionen der SPD und Bündnis 90/Die Grünen in der Bremer Bürgerschaft 2007, S. 3). Als Mindestlohn wird damit de facto die unterste Lohngruppe des Tarifvertrages für den öffentlichen Dienst (TVöD) bzw. des Tarifvertrages für die Länder (TV-L) bestimmt, die derzeit (März 2008) bei $1.286 €$ bzw. $1.325 €$ pro Monat liegt, was bei einer 39Stunden-Woche einem Stundenmindestlohn von 7,60€ bzw. 7,84 € entspricht. Die Mindestlohnregelung greift jedoch nur bei Branchen ohne „maßgeblichen Tarifvertrag", während Branchen mit niedrigeren Tariflöhnen hiervon unberührt bleiben.

Eine weitergehende Regelung findet sich im neu gefassten Berliner Vergabegesetz, das hierzu Folgendes ausführt: „Sofern für einzelne Branchen in Berlin keine Entgelttarife bestehen oder die in Berlin bestehenden und im konkreten Fall anwendbaren Entgelttarife ein Entgelt von weniger als 7,50€ je Stunde vorsehen, erfolgt die Vergabe mit der Auflage, dass die Unternehmen ihre Arbeitnehmerinnen und Arbeitnehmer (ohne Auszubildende) bei der Ausführung dieser Leistungen mit mindestens $7,50 €$ je Stunde entlohnen" (Berliner Senat 2008, S. 4). Im Gegensatz zu Bremen gilt in Berlin der als Vergabekriterium festgelegte Mindestlohn von 7,50€ auch dann, wenn in Tarifverträgen niedrigere Löhne vereinbart wurden.

Über die rechtliche Zulässigkeit der Einführung eines Mindestlohns als soziales Vergabekriterium wird derzeit heftig gestritten. Die Vereinigung der Unternehmensverbände in Berlin und Brandenburg vertritt die Auffassung, dass es sich bei der Berliner Mindestlohnregelung um einen verfassungswidrigen Eingriff in die Tarifautonomie handelt und behält sich die Möglichkeit einer Klage gegen das Tarifgesetz vor (UVB 2008). Demgegenüber kommt ein Gutachten des Wissenschaftlichen Dienstes des Deutschen Bundestages zu dem Ergebnis, dass in Anlehnung an das Urteil des Bundesverfassungsgerichtes zur Tariftreue auch eine Mindestlohnregelung im Vergaberecht verfassungskonform ist (Schneider-Schahn/ Sahl 2007).

\section{$P$ \\ Fazit}

Die politischen Auseinandersetzungen um Tariftreueregelungen haben in den letzten Jahren wieder deutlich an Dynamik gewonnen. Dies gilt sowohl quantitativ in Hinblick auf die wachsende Zahl von Bundesländern mit eigenen Tariftreuegesetzen als auch qualitativ in Hinblick auf die sektorale Reichweite dieser Gesetze. Angesichts der hohen ökonomischen Bedeutung des öffentlichen Auftragswesens nutzt der Staat hier seine Marktmacht, um zur Stabilisierung des deutschen Tarifvertragssystems beizutragen.

Die bestehenden Tariftreuegesetze weisen allerdings immer noch große Regelungslücken auf und bilden aus gesamtdeutscher Perspektive einen bunten Flickenteppich. Im Rahmen der aktuell diskutierten Revision des bundesdeutschen Vergaberechts hätte die Bundesregierung eine gute Gelegenheit, einen neuen Anlauf für ein bundesweit einheitliches Tariftreuegesetz zu nehmen. ${ }^{3}$ Ein solches Gesetz sollte dem aktuellen Berliner Beispiel folgen und die Beschränkung auf einzelne Bran-

\footnotetext{
3 Immerhin hat der SPD-Vorsitzende Kurt Beck hierzu auf dem letzten ver.di Kongress erklärt: „Nach meiner Überzeugung ist es unverzichtbar, dass wir überall dort, wo Aufträge der Öffentlichen Hand vergeben werden, über Tariftreue-Gesetze dafür sorgen, dass nur diejenigen Aufträge der Städte und Kommunen, der Länder und des Bundes (!) und ihrer Gesellschaften bekommen, die den jeweiligen anständigen Tariflohn bezahlen" (Beck 2007).
} 
chen zugunsten universeller Geltung aufgeben. Außerdem ist - so lange Deutschland noch über keinen allgemeinen branchenübergreifenden Mindestlohn verfügt - eine Verknüpfung von Tariftreue und Mindestlohn sinnvoll.

Nach dem aktuellen Urteil des Europäischen Gerichtshofes ist die Zukunft der Tariftreueregelungen insgesamt jedoch mehr als ungewiss. Sollte es nicht gelingen, doch noch einen europarechtskonformen Weg zur Absicherung von Tariftreuregelungen zu finden, so käme es zu einem rigorosen Lohnkostenwettbewerb, bei dem der Staat gezwungen wäre, das „,wirtschaftlichste" Angebot anzunehmen und damit nicht-tarifgebundene Unternehmen strukturell bevorteilen müsste. Entgegen der ursprünglichen Intention von Tariftreuegesetzen würde der Staat das Tarifvertrags- system dann nicht stabilisieren, sondern im Gegenteil selbst noch zu seiner weiteren Erosion beitragen. In diesem Fall wären als alternative Regelungen nicht nur die Einführung eines allgemeinen gesetzlichen Mindestlohns, sondern auch eine Reform, die die Allgemeinverbindlichkeit von Tarifverträgen deutlich erleichtert, immer dringlicher (Zachert 2004).

\section{LITERATUR}

Bechtolsheim, C. von/Betz, K. (2006): Berücksichtigung von Entlohnungskriterien bei Vergabeentscheidungen, Stellungnahme im Auftrag der Dienstleistungsgewerkschaft ver.di, Berlin, 15. November Beck, K. (2007): Rede auf dem ver.di Kongress in Leipzig, 5. Oktober (http://bundeskongress2007.verdi.de/reden_berichte/kurt_beck) Berliner Senat (2008): Erstes Gesetz zur Änderung des Berliner Vergabegesetzes, Vorlage zur Beschlussfassung, Abgeordnetenhaus Berlin, Drucksache 16/1155, 5. Februar

Bispinck, R./Schulten, T. (2008): Aktuelle Mindestlohndebatte: Branchenlösungen oder gesetzlicher Mindestlohn? in: WSI-Mitteilungen 3, S. 151-158

Bundesministerium für Wirtschaft und Technologie (BMWi) (2007): Öffentliches Beschaffungswesen, Gutachten des Wissenschaftlichen Beirates des BMWI, Dezember

Bot, Y. (2007): Schlussanträge des Generalanwalts beim Europäischen Gerichtshof in der Rechtsache C-346/06, 20. September

Bundesverfassungsgericht (2006): Beschluss vom 1. Juli 2006, 1BvL 4/00, www.bundesverfassungsgericht.de/entscheidungen/ls20060711_ 1bvl000400.html

Burgess, P. (2006): Living Wage-Kampagnen in London, in: Sterkel, G. et al. (Hrsg.): Mindestlöhne gegen Lohndumping, Hamburg, S. 243-256 Butollo, F. (2007): FAIRgabe in Berlin? Für eine faire, ökologische und soziale öffentliche Auftragsvergabe, hrsg. von WEED (Weltwirtschaft, Ökologie \& Entwicklung), Berlin, Dezember Europäische Kommission (2001): Mitteilung der Kommission über die Auslegung des gemeinschaftlichen Vergaberechts und die Möglichkeiten zur Berücksichtigung sozialer Belange bei der Vergabe öffentlicher Aufträge, $\operatorname{KOM(2001)} 566$ endgültig, Brüssel, 15. Oktober

Europäische Union (2004): Richtlinie 2004/18/EG des Europäischen Parlaments und des Rates vom 31. März über die Koordinierung der Verfahren zur Vergabe öffentlicher Bauaufträge, Lieferaufträge und Dienstleistungsaufträge, in: Amtblatt der Europäischen Union L 134, 30. April, S. 115-240

Europäischer Gerichtshof (2008): Urteil des Gerichtshofes in der Rechtssache C-346/06 (Tariftreueregelung in Niedersachsen), 3. April
European Commission (2004): A Report on the Functioning of Public Procurement Markets in the EU: Benefits from the Application of EU Directives and Challenges for the Future, Februar (http://ec.europa.eu/ internal market/publicprocurement/docs/public-proc-market-finalreport en.pdf)

Fraktionen der SPD und Bündnis 90/Die Grünen in der Bremer Bürgerschaft (2007): Änderung des Vergabegesetzes für das Land Bremen, Bremische Bürgerschaft Drucksache 17/156, 27. November

Greater London Authority (2007): A Fairer London: The Living Wage in London, London, März

Hamburger Senat (2007): Evaluierungsbericht zum Hamburgischen Vergabegesetz (HmbVgG), Mitteilung des Senates an die Bürgerschaft, Bürgerschaft der Freien und Hansestadt Hamburg Drucksache 18/7388, 20. November

Handler, H. (2005): Das öffentliche Auftragswesen im gesamtwirtschaftlichen Zusammenhang, WIFO Working Papers 250, April

Leinemann, R. (2007): Die Vergabe öffentlicher Aufträge, Köln

McCrudden, C. (2004): Using Public Procurement to achieve Social Outcomes, in: Natural Resources Forum 4, S. 257-267

Pollin, R./Brenner, M. D./Wicks-Lim, J./Luce, S. (2008): A Measure of Fairness: The Economics of Living Wages and Minimum Wages in the United States, Ithaca

Schneider-Schahn, T./Sahl, J. C. (2007): Mindestlöhne über Vergaberecht. Zulässigkeit von Mindestlöhnen als Kriterium im Vergabeverfahren. Wissenschaftlicher Dienst des Deutschen Bundestages WD 7 - 121/07

Stefaniak, A./Vollmer, H. (2005): Evaluierung Tariftreuegesetz NordrheinWestfalen - Endbericht, Dortmund, Februar

Vereinigung der Unternehmensverbände in Berlin und Brandenburg (UVB) (2008): Stellungnahme zur Beschlussfassungsvorlage über ein erstes Gesetz zur Änderung des Berliner Vergabegesetzes, 20. Februar Zachert, U. (2004): Allgemeinverbindlicherklärung, in: Peter, G./Kempen, O. E./Zachert, U.: Die Sicherung tariflicher Mindeststandards, Baden-Baden, S. 11-32 